\title{
Quadrature Formulas Involving Derivatives of the Integrand
}

\author{
By Preston C. Hammer and Howard H. Wicke
}

1. Introduction. In this paper we demonstrate the existence of quadrature for mulas of the following types. For $k$ an odd positive integer,

$$
\int_{1}^{1} f(x) d x=2 \sum_{i=0}^{(k-1) / 2} \frac{f^{(2 i)}(0)}{(2 i+1) !}+\sum_{j=1}^{m} a_{j}\left[f^{(k)}\left(x_{j}\right)-f^{(k)}\left(-x_{j}\right)\right]+R_{m, k}(f) .
$$

For $k$ an even positive integer,

$$
\int_{1}^{1} f(x) d x=2 \sum_{i=0}^{(k-2) / 2} \frac{f^{(2 i)}(0)}{(2 i+1) !}+\sum_{j=1}^{m} a_{j}\left[f^{(k)}\left(x_{j}\right)+f^{(k)}\left(-x_{j}\right)\right]+R_{m, k}(f) .
$$

Each of formulas (1) and (2) will be shown to exist so that they are exact for polynomials of degree at most $4 m+k$ for odd $k$, and $4 m+k-1$ for even $k$.

It is possible to use tables published by Hammer, Marlowe, and Stroud [2] and extended by Fishman [1] to obtain formulas of the form

$$
\int_{0}^{1} f(x) d x=f(1)+\cdot \sum_{j=1}^{m} a_{j} f^{\prime}\left(x_{j}\right)+R_{m}(f)
$$

and likewise formulas using higher derivatives. These formulas are asymmetrical and for some uses would be more appropriate than formula (1) or (2).

It is considered that formula (1), (2), or (3) may be useful when the integrand function is represented as a variable integral for which derivatives may be easier to compute than the integrand function itself. It is also anticipated that these formulas may be used in the numerical solution of differential equations.

Kopal [3] has devised formulas using the first derivative values. His approach leaves difficulties in establishing existence and reality of evaluation points and in some cases he has computed more than one formula of the same degree. The method we propose has direct connection with the established theory of orthogonal polynomials which gives the existence, reality, and distinctness of the evaluation points and the positiveness of the weights $a_{j}$. A linear transformation to give integration limits $-h, h$ in formula (1) or (2) results in multiplying each derivative of order $n$ by $h^{n+1}$.

We have not identified the orthogonal polynomial systems with any treated in detail in the literature. However, tables of the $a_{j}$ and $x_{j}$ for $k=1,2, m=1$ (1) 10 , and $k=3,4, m=1$ (1) 9 , have been computed by G. W. Struble in [5], where tables for the $\mu_{m, k}^{(m)}$ of the remainder terms are also to be found. For purposes of computation we give a standard type recursion formula permitting generation of each polynomial in a sequence from its two predecessors.

Throughout the paper we assume that the integrand function has all-order derivatives appearing and that these are continuous.

Received April 16, 1959; in revised form, July 9, 1959. This work is supported in part by the Office of Ordnance Research, U. S. Army. 
2. Reduction of the Problems. It is well known and readily established that

$$
\int_{0}^{1}\left(\int_{0}^{x}\right)^{n} g(x)(d x)^{n+1}=\frac{1}{n !} \int_{0}^{1}(1-x)^{n} g(x) d x
$$

where $\left(\int_{0}^{x}\right)^{n} g(x)(d x)^{n}$ denotes the result of integrating $g(x)$ successively $n$ times over $(0, x)$. Now our formulas (1) and (2), by choice of form, will hold for every odd integrand function $f(x)$ and hence we write $f(x)=f_{0}(x)+f_{1}(x)$, where

$$
f_{0}(x)=\frac{f(x)+f(-x)}{2} \text { and } f_{1}(x)=\frac{f(x)-f(-x)}{2} .
$$

The functions $f_{0}$ and $f_{1}$ may be called the even and odd components of $f$, respectively. We now observe

$$
\int_{-1}^{1} f(x) d x=2 \int_{0}^{1} f_{0}(x) d x \text { and } f_{0}{ }^{(k)}(x)=\frac{f^{(k)}(x)+(-1)^{k} f^{(k)}(-x)}{2} .
$$

Moreover,

$$
\begin{aligned}
& \int_{0}^{1} f_{0}(x) d x= \int_{0}^{1}\left(\int_{0}^{x}\right)^{k} f_{0}^{(k)}(x)(d x)^{k+1}+S_{k}(f), \text { where } \\
& S_{k}(f)= \begin{cases}\sum_{i=0}^{(k-1) / 2} \frac{f^{(2 i)}(0)}{(2 i+1) !}, & \text { for } k \text { odd } \\
\sum_{i=0}^{(k-2) / 2} \frac{f^{(2 i)}(0)}{(2 i+1) !}, & \text { for } k \text { even. }\end{cases}
\end{aligned}
$$

Hence our formulas (1) and (2) may be written, using formulas (4), (5), and (6), and dropping $2 S_{k}(f)$ from each side, as follows:

$$
\frac{2}{k ! !} \int_{0}^{1}(1-x)^{k} f_{0}{ }^{(k)}(x) d x=2 \sum_{j=1}^{m} a_{j} f_{0}{ }^{(k)}\left(x_{j}\right)+R_{m, k}(f) \text {. }
$$

Now we only need derive formulas (7) exact for even-degree polynomials replacing $f_{0}(x)$. Then, if $k$ is odd we have with $u=x^{2}$

$\frac{2}{k !} \int_{0}^{1}(1-x)^{k} x P\left(x^{2}\right) d x=\frac{1}{k !} \int_{0}^{1}(1-\sqrt{u})^{k} P(u) d u$

$$
=2 \sum_{j=1}^{m} a_{j} \sqrt{u_{j}} P\left(u_{j}\right), \text { and thus, }
$$

$$
\frac{1}{k !} \int_{0}^{1}(1-\sqrt{u})^{k} P(u) d u=\sum_{j=1}^{m} b_{j} P\left(u_{j}\right), \quad \text { where } b_{j}=2 a_{j} \sqrt{u_{j}} .
$$

On the other hand, if $k$ is even, so that $f_{0}^{(k)}(x)$ is replaced by an even polynomial $P\left(x^{2}\right)$, then we have with $u=x^{2}, b_{j}=2 a_{j}$,

$$
\frac{1}{k !} \int_{0}^{1} \frac{(1-\sqrt{u})^{k}}{\sqrt{ } u} P(u) d u=\sum_{j=1}^{m} b_{j} P\left(u_{j}\right) .
$$

Now formulas (8) and (9) are in standard form to apply the theory of orthogonal polynomials. The weight functions are positive-valued and appropriate. Hence there exists a sequence $\left\{P_{m, k}(u)\right\}$ of orthogonal polynomials for each $k=1$, 
$2, \cdots$, the zeros of which are the squares of the proposed evaluation points $x_{j}$, and positive numbers $b_{j}$ from which the $a_{j}$ are determined. The zeros are real, distinct, and in the interval.

The weight functions are simple in form but we have been unable to find references in which this class has been treated. For $k=1$ we have verified that the system of polynomials is not a classical one since $\left\{P_{m, 1}^{\prime}(u)\right\}$ is not orthogonal. For purposes of calculating formulas, however, we state in the next section recursion formulas which permit sequential generation of the polynomials for each $k \geqq 1$.

3. Recursion Formula for Polynomials. The recursion formula stated here is of the standard sort, but its form is preferable to the one given by Szegö [4], p. 41, since it involves only the coefficients of $P_{n-1}$ and $P_{n-2}$ to calculate the multipliers $B_{n}$ and $C_{n}$. This is important when explicit formulas for the multipliers have not been determined. We assume $\left\{P_{n}(u)\right\}$ is a system of polynomials with leading coefficient 1 , orthogonal over the interval $[a, b]$ with respect to the weight function $w(u)$. Then we have

$$
P_{n}(u)=\left(u+B_{n}\right) P_{n-1}(u)-C_{n} P_{n-2}(u) . \quad n \geqq 2
$$

If we define

$$
\mu_{n}{ }^{(k)}=\int_{a}^{b} w(u) u^{k} P_{n}(u) d u
$$

we may observe $\mu_{n}{ }^{(k)}=0,0 \leqq k<n$, and

$$
\mu_{n}^{(n)}=\int_{a}^{b} w(u) u^{n} P_{n}(u) d u=\int_{a}^{b} w(u)\left(P_{n}(u)\right)^{2} d u .
$$

We also define $c_{n-1}^{(j)}$ as the coefficient of $u^{j}$ in $P_{n-1}(u)$. Then $B_{n}$ and $C_{n}$ are given by

$$
\begin{gathered}
B_{n}=-\left(c_{n-1}^{(n-2)}+\frac{\mu_{n-1}^{(n)}}{\mu_{n-1}^{(n-1)}}\right) \\
C_{n}=\frac{\mu_{n-1}^{(n-1)}}{\mu_{n-2}^{(n-2)}} .
\end{gathered}
$$

For our particular problem, the $\mu_{n-2}^{(n-2)}, \mu_{n-1}^{(n-1)}$, and $\mu_{n-1}^{(n)}$ are linear combinations of the coefficients of $P_{n-2}$ and $P_{n-1}$ with rational multipliers and the coefficients of every $P_{n}$ are rational. Hence, in the absence of better formulas, starting with $P_{n}$ and $P_{1}$, we can generate the remaining polynomials, in principle. In practice, of course, the coefficients grow very rapidly in exact rational form.

Now $P_{0, k}=1$ for every $k$, and, for degree one, $\left\{P_{1, k}(u)\right\}$ is given by

$$
P_{1,2 n-1}(u)=u-\frac{3}{(2 n+3)(n+1)}, \quad n=1,2,3, \cdots
$$

and

$$
P_{1,2 n}(u)=u-\frac{1}{(2 n+3)(n+1)}, \quad n=1,2,3, \cdots .
$$

Hence, with the recursion formula (10), successive higher-degree polynomials may be calculated. 
The orthonormal sequence $\left\{Q_{n}(u)\right\}$ is determined by

$$
P_{n}(u)=\sqrt{\mu_{n}^{(n)}} Q_{n}(u) \text {. }
$$

4. Remainders for the Integration Formulas. In this section we give the remainder formulas. We will give the explicit functions to which Rolle's Theorem may be applied. The method is a variation of Markoff's for Hermite (osculating) interpolation (Szegö [4], p. 369.) As usual, we obtain the highest-degree polynomial $H(x)$ for which the formula is exact and which agrees with the integrand function or its derivatives at all evaluation points. Then we estimate

$$
f^{(k)}(x)-H^{(k)}(x)
$$

and $f_{0}^{(k)}(x)-H_{0}^{(k)}(x)$, and use formula $(7)$ to find $R_{m, k}(f)$. (Here $H_{0}$ is the even component of $H$.)

For $k$ odd and $2 m+1$ evaluation points $0, \pm x_{1}, \cdots, \pm x_{m}$, we require

$$
H(0)=f(0), \quad H^{(n)}(0)=f^{(n)}(0)
$$

for $n=1, \cdots, k, H^{(k)}\left( \pm x_{j}\right)=f^{(k)}\left( \pm x_{j}\right), H^{(k+1)}\left( \pm x_{j}\right)=f^{(k+1)}\left( \pm x_{j}\right), j=1$, $\cdots, m$. Then $H(x)$ is a polynomial of degree at most $4 m+k$. Let $P_{m}\left(x^{2}\right)$ be the polynomial $P_{m, k}(u)$ determining the evaluation points $x_{j}$ by $P_{m}\left(x_{j}{ }^{2}\right)=0, j=1$, $\cdots, m$. Then let $x$ be any number which is none of $0, \pm x_{j}$. Define

$$
F(z)=f^{(k)}(z)-H^{(k)}(z)-\frac{f^{(k)}(x)-H^{(k)}(x)}{x\left[P_{m}\left(x^{2}\right)\right]^{2}} z\left[P_{m}\left(z^{2}\right)\right]^{2}
$$

Then $F(z)$ vanishes at $x, 0, \pm x_{j}(2 m+2$ distinct points $)$. Also, $F^{\prime}(z)$ vanishes at $\pm x_{j}$ and $2 m+1$ other distinct points, or $4 m+1$ points. Hence, applying Rolle's Theorem there exists a $\xi_{1}$ such that $F^{(4 m+1)}\left(\xi_{1}\right)=0$, and thus

$$
f^{(k)}(x)-H^{(k)}(x)=\frac{f^{(4 m+k+1)}\left(\xi_{1}\right) x\left[P_{m}\left(x^{2}\right)\right]^{2}}{(4 m+1) !} .
$$

From equation (18) applied to $x$ and $-x$, and from the continuity of $f^{(4 m+k+1)}(x)$, it follows that the even components satisfy

$$
f_{0}{ }^{(k)}(x)-H_{0}{ }^{(k)}(x)=\frac{f^{(4 m+k+1)}\left(\xi_{2}\right) x\left[P_{m}\left(x^{2}\right)\right]^{2}}{(4 m+1) !} .
$$

Hence, from equation $(\tau)$ by the continuity of $f^{(4 m+k+1)}(x)$ and the first theorem of the mean for integrals, we have, with $u=x^{2}$, and for some $\eta \in[-1,1]$,

$$
R_{m, k}(f)=\frac{f^{(4 m+k+1)}(\eta)}{(4 m+1) !} \int_{0}^{1} \frac{(1-\sqrt{u})^{k}}{k !}\left[P_{m}(u)\right]^{2} d u, \quad \text { for } k \text { odd },
$$

or replacing the integral by $\mu_{m, k}^{(m)}$,

$$
R_{m, k}(f)=\frac{f^{(4 m+k+1)}(\eta)}{(4 m+1) !} \mu_{m, k}^{(m)}, \quad \text { for } k \text { odd }
$$

For $k$ even, we require $H(x)$, of at most degree $4 m+k-1$, such that

$$
H(0)=f(0), \quad H^{(n)}(0)=f^{(n)}(0),
$$

for $n=1, \cdots, k-1, H^{(k)}\left( \pm x_{j}\right)=f^{(k)}\left( \pm x_{j}\right), H^{(k+1)}\left( \pm x_{j}\right)=f^{(k+1)}\left( \pm x_{j}\right)$, for 
for $j=1, \cdots, m$. Then, for $x$ not equal to one of $\pm x_{j}$, we define

$$
F(z)=f^{(k)}(z)-H^{(k)}(z)-\frac{f^{(k)}(x)-H^{(k)}(x)}{\left[P_{m}\left(x^{2}\right)\right]^{2}}\left[P_{m}\left(z^{2}\right)\right]^{2} .
$$

Now $F^{\prime}(z)$ vanishes at $4 m$ distinct points, and there exists a $\xi_{1}$ such that

$$
F^{(4 m)}\left(\xi_{1}\right)=0,
$$

which implies

$$
f^{(k)}(x)-H^{(k)}(x)=\frac{f^{(4 m+k)}\left(\xi_{1}\right)\left[P_{m}\left(x^{2}\right)\right]^{2}}{(4 m) !} .
$$

The even components $f_{0}$ and $H_{0}$ then satisfy

$$
f_{0}^{(k)}(x)-H_{0}{ }^{(k)}(x)=\frac{f^{(4 m+k)}\left(\xi_{2}\right)\left[P_{m}\left(x^{2}\right)\right]^{2}}{(4 m) !},
$$

using the same argument used in the passage from (18) to (19). Then, as before, substitution in equation ( 7 ) gives, with $u=x^{2}$, and for some $\eta \in[-1,1]$

$$
R_{m, k}(f)=\frac{f^{(4 m+k)}(\eta)}{(4 m) !} \int_{0}^{1} \frac{(1-\sqrt{u})^{k}}{k ! \sqrt{u}}\left[P_{m}(u)\right]^{2} d u, \quad \text { for } k \text { even, }
$$

or, with the integral replaced by $\mu_{m, k}^{(m)}$,

$$
R_{m, k}(f)=\frac{f^{(4 m+k)}(\eta)}{(4 m) !} \mu_{m, k}^{(m)}, \quad \text { for } k \text { even. }
$$

The number $\mu_{m, k}^{(m)}$ may be determined simply in the low-degree cases by substituting in the numerical formula integrands $x^{4 m+k+1}$ if $k$ is odd, and $x^{4 m+k}$ if $k$ is even.

It may be noted that our explicit choice of $H$ is made for convenience in each case. That is, formulas (1) and (2) hold with $f=H$ and $R_{m k}=0$, and $H$ is a highest-degree polynomial for which this is true-i.e., no higher even power of $x$ may be included. Moreover, in formula (6) we have

$$
\int_{0}^{1} H_{0}(x) d x=\int_{0}^{1}\left(\int_{0}^{x}\right)^{k+1} H_{0}^{(k)}(x)(d x)^{k+1}+S_{k}(f),
$$

since $f^{(j)}(0)=H^{(j)}(0)$, for appropriate values of $j$. Numerical tables of $a_{j}, x$, and the constant in the remainder have been calculated by G. W. Struble [5] for small values of $k$.

University of Wisconsin Madison, Wisconsin and Sandia Corporation Albuquerque, New Mexico

1. H. Fishman, "Numerical integration constants," MTAC, v. 11, 1957, p. 1.

2. P. C. HAMmer, O. J. MarLOWE, \& A. H. Stroud, "Numerical integration over simplexes and cones," MTAC, v. 10, 1956, p. 130-137.

3. Z. KOPAL, Numerical Analysis, Wiley \& Sons, New York, 1956.

4. G. SzEGö, "Orthogonal polynomials," Amer. Math. Soc., Colloquium Publs., v. 23, 1939.

5. G. W. STRUBLE, "Quadrature formulas using values of derivatives," Math. Comp. $(M T A \dot{C})$, v. 14,1960, p. 8-12. 